\title{
The Relation between Anatomical Variations of Osteomeatal Complex \& Nasal Structures and Chronic Sinusitis by Computed Tomography
}

\author{
Naglaa M. Elsayed ${ }^{1,2, ~ *}$, Lujain F. Abdalaal ${ }^{1}$ \\ ${ }^{1}$ Diagnostic Radiology Department, Faculty of Applied Medical Sciences, KAU, Jeddah, KSA \\ ${ }^{2}$ Diagnostic Radiology Department, Faculty of Medicine, Cairo University, Cairo, Egypt
}

Email address:

nmmalsayed@kau.edu.sa (N. M. Elsayed), naglaamostafaelsayed@yahoo.com (N. M. Elsayed), lujain.a@hotmail.com (L. F. Abdalaal)

\section{To cite this article:}

Naglaa M. Elsayed, Lujain F. Abdalaal. The Relation between Anatomical Variations of Osteomeatal Complex \& Nasal Structures and Chronic Sinusitis by Computed Tomography. International Journal of Medical Imaging. Vol. 3, No. 2, 2015, pp. 16-20.

doi: $10.11648 /$ j.ijmi.20150302.12

\begin{abstract}
Background: The anatomy of the nose and paranasal sinuses is complex, and many anatomical variations have been thoroughly studied. There is debate about the effect of these variations on the occurrence and severity of sinusitis. Computed Tomography (CT) scan is the modality of choice for evaluation of variable anatomical variations and different forms of sinusitis. Aim: The aim of this research was to study the relation between recurrent sinusitis and anatomical variations of the osteomeatal complex and nasal structures by CT. Patients and Methods: A cross section observation study was performed. We collected data of 89 patients subjected to CT of the paranasal sinuses. CT was performed using SIEMENS SOMATOM SENSATION dual source machine. Data analysis was performed using SPSS program, version (19.0.0): Results: All patients in the study population showed one or more anatomical variations and only $85.3 \%$ of them had sinusitis. Infundibular pattern of sinusitis had the highest incidence and the lowest patterns were sphenoethmoid recess and sporadic unclassified patterns. Anatomical variations found by CT, nasal septal deviation represented the most common variation (50\%). Conclusion: There was a statistically significant correlation between nasal septal deviation, uncinate process anomalies and some patterns of sinusitis with the $\mathrm{p}$ value $=<0.05$.
\end{abstract}

Keywords: Paranasal Sinuses, Osteomeatal Complex, Sinusitis, CT, anatomical Variation

\section{Introduction}

There is a wide range of anatomical variations of the nose, paranasal sinuses (PNS) and osteomeatal complex best detected by Computed Tomography (CT), which is a noninvasive imaging modality with high image resolution and reconstruction capability. These variations may lead to impaired mucociliary drainage of the PNS results in sinusitis. Inflammatory changes of the PNS are radiologically grouped into various patterns; infundibular, ostiomeatal unit (OMU), spheno-ethmoid recess, sinonasal polyposis of the nose and PNS, sporadic unclassified patterns e.g. mucocele and nonspecific mucosal thickening[1,2,3]. Many anatomical variations have been studied and the correlation between them and sinusitis in not clear up till now [4].Some studies showed statistically significant association between common anatomic variations and the presence of sinusitis [5]. While in other studies, no statistically significant correlation was found[6].

The aim of this study was to study the possible correlation of anatomical variations of the nose, PNS and osteomeatal complex to chronic sinusitis by means of C.T.

\section{Patients and Methods}

\subsection{Type of Study}

A cross section observation study was done at the diagnostic radiology department, KAUH after obtaining research ethical approval from the medical research ethical committee, Faculty of medicine, KAU.

\subsection{Patients}

This study included 89 patients subjected to CT of the PNS 
done between November, 2012 and March, 2013, who had signs and symptoms of chronic sinusitis - as diagnosed by the referral ENT doctors - e.g. facial pain and tenderness over PNS, nasal congestion, headache, post nasal discharge, night irritating cough and reduced smell sensation lasting for more than 3 continuous months. Patient's consent was obtained prior CT examination.

Participants included 42 females and 47 males ranging in age from 20 to 72 years with the median age is 46 years.

\subsection{Technique of CT}

Non contrast CT was performed using SIEMENS SOMATOM SENSATION dual source machine. Axial planes were done for all patients in the supine position, and then images were sent to the workstation for coronal and sagittal reconstruction.

\subsection{Image Interpretation}

CT scan of each patient was reviewed by two expert radiologists minded by the patients symptoms of sinusitis for the presence of evidences of sinusitis e.g. mucosal thickening, sinus opacification, polyp or mucocele, in addition to the presence of anatomical variations of PNS including concha bullosa, turbinate hypertrophy, Agger nasi cell, Haller's cells, pneumatized uncinate process, osteomeatal complex, septal deviation or maxillary, sphenoid or frontal hypoplasia.Inflammatory changes of the PNS were classified into five patterns; 1- Infundibular pattern 2- Ostiomeatal unit pattern 3- Spheno-ethmoid recess pattern 4- Sinonasal polyposis 5- Sporadic unclassified pattern and pansinusitis.

\subsection{Statistical Analysis}

Demographic data of participants together with CT findings were collected and statistically analyzed using the

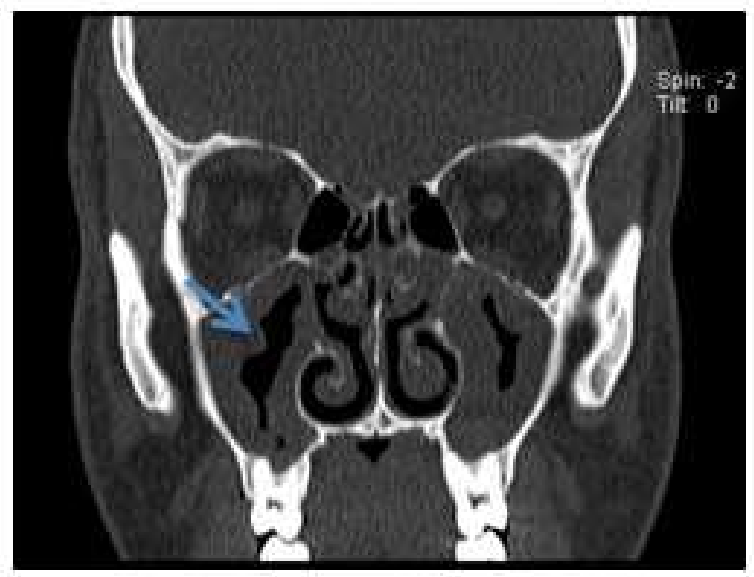

A
SPSS program version 19.0.0 (SPSS, Inc. Chicago, USA) software package. To correlate the incidence of sinusitis with different anatomical variations of osteomeatal complex and nasal cavity, Chi-square test or Fischer's exact test -when appropriate- were used to assess the significance of the correlation between anatomical variations of osteomeatal complex and sinusitis. Differences in all testes with a p-value of $<0.05$ is considered to be statistically significant.

\section{Results}

All participants showed one or more anatomical variations, and out of them, $85.3 \%$ had sinusitis. The distribution of different patterns of sinusitis among the study population is shown in (Fig. 1) where infundibular pattern had the highest incidence $(28 \%)$, followed by sinonasal polyposis and pansinusitis (20\% for each) (Fig. 2), then OMU (14\%). The least patterns were sphenoethmoid recess pattern and sporadic unclassified pattern ( $9 \%$ for each). Unclassified pattern included nonspecific mucosal thickening or and mucoceles. (Fig.3)

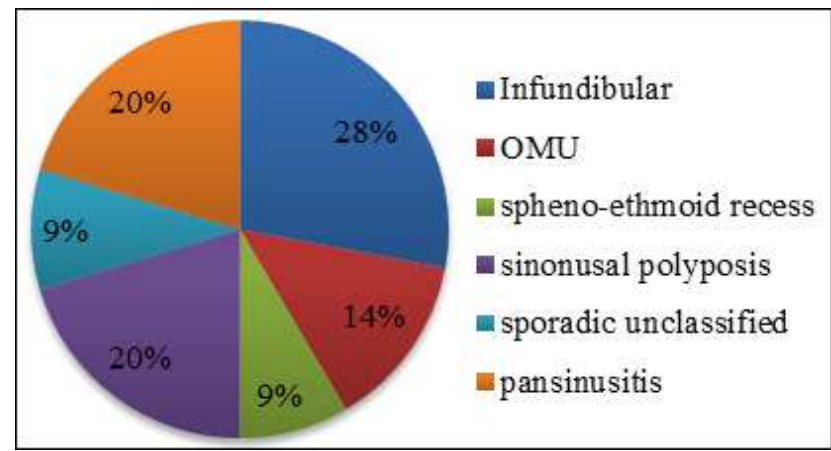

Fig. 1. The distribution of different patterns of sinusitis among the study group.

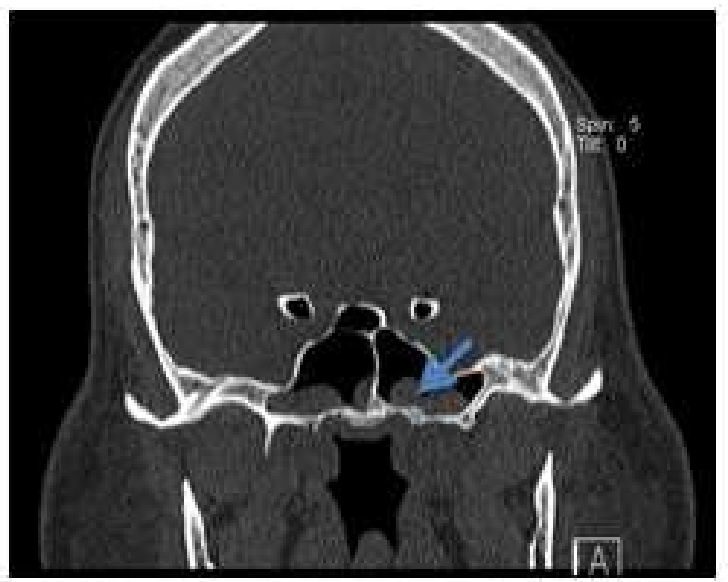

B

Fig. 2. A\& B: Coronal CT shows bilateral maxillary and sphenoid polyps (arrows). Sinonasal polyposis pattern. 


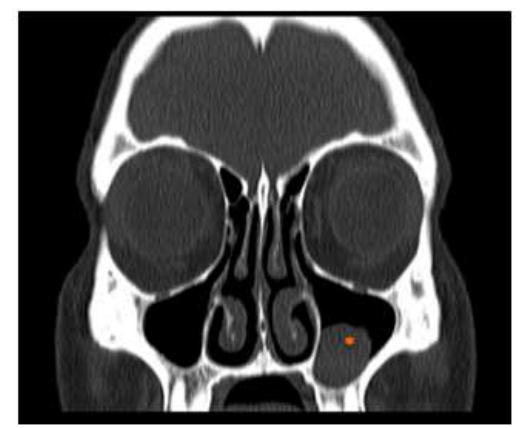

Fig. 3. CT coronal image shows mucous retention cyst in the left maxillary sinus (*). Sporadic unclassified sinusitis.

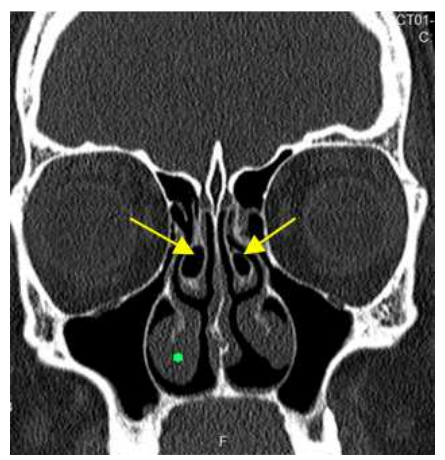

Fig. 4. CT coronal image shows bilateral concha bullosa (arrows). Notice the bilateral inferior turbinate hypertrophy (*).

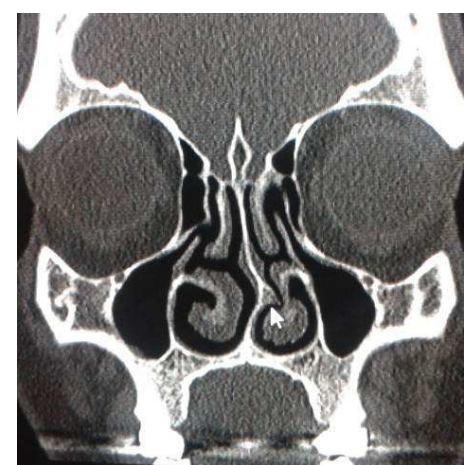

Fig. 5. CT coronal image shows deviated nasal septum with bony spur (arrow).

Table 1. The relation between different patterns of sinusitis and anatomical variations encountered in the study population. Notice that some patients have more than one form of sinusitis.

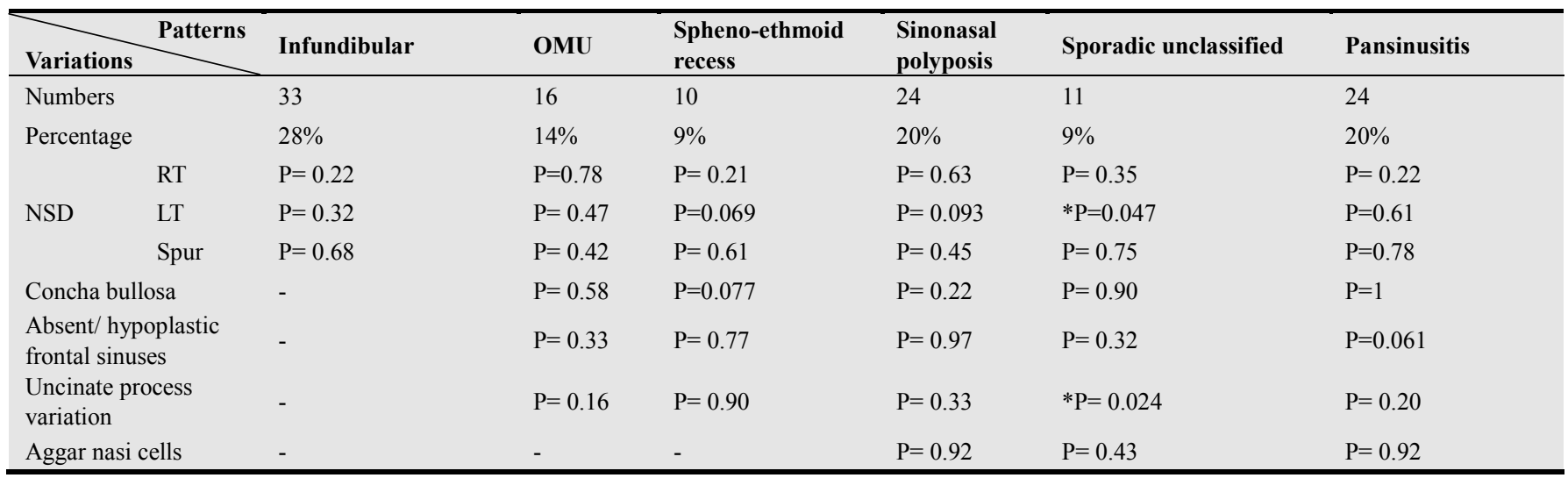

* Statistically significant
One or more anatomical variations were seen by $\mathrm{CT}$ in all participants. Nasal septum deviation (NSD) represented the most commonly encountered variation $(50 \%)$ with the right and left sides are almost equal $(26 \%$ and $24 \%$ respectively). This is followed by concha bullosa and nasal septum spur (16\% for each) (Fig. 4 and 5$)$.

Absent/hypoplastic frontal sinus represented (9\%). Uncinate process variations (deviation or aeration) represented (6\%) and Agger nasi cell was seen in only 3\%. The detailed distribution of common anatomical variations of the nose and PNS among the study population is seen in figure (6).

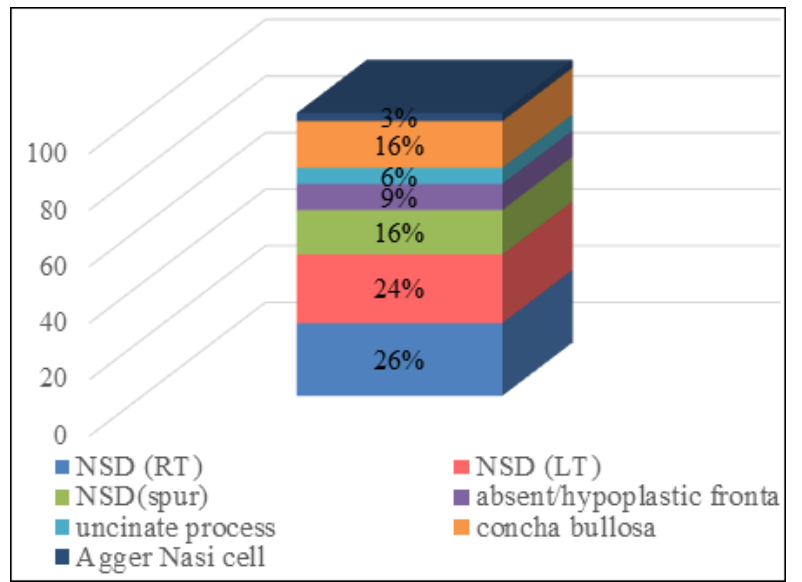

Fig. 6. Distribution of anatomical variations of the nasal structures and osteomeatal complex among the study group.

Statistical analysis correlation between different patterns of sinusitis and anatomical variation of the nose and PNS was done using Chi-square/ Fischer exact test. Table1shows different patterns of sinusitis and the incidence of associated anatomical variations. Considering the statistical significance of $p$ value $<0.05$, there was statistically significant correlation between sporadic unclassified sinusitis and both nasal septaum deviation and uncinate process variations ( $\mathrm{p}$ value $=0.047$ and 0.024 respectively). 


\section{Discussion}

Some of our results match with those mentioned in literatures and others don't match. Infundibula pattern (the commonest pattern of sinusitis (28\%) in the current study) is more or less the same of Babbel et al, 1991 where the infundibular pattern is the most common detected pattern in his study (26\%) [7]. Also, sphenoethmoid recess pattern has the least incidence $(9 \%)$, which is in concordance with the results of Zinreich S, et al, 2003 where sphenoethmoidal recess representing only $13.6 \%[2]$. On the other hand, some patterns are seen less frequently than mentioned in literatures $[3,8]$. Ostiomeatal unit pattern is seen in $14 \%$ and sporadic unclassified pattern is seen in only $9 \%$ of our study group. These results denote the great variation in the incidences of different patterns of sinusitis. In the current study, anatomical variations of the PNS are surprisingly found in all population study. This incidence is very high compared to other studies. In studies of Adeel M, 2013 with a sample size of 77, 88.5\% of the population study had anatomical variation and in another study - but with smaller sample size (40 patients), the incidence was much less (51.9\%) [9]. The incidence of these variations differs from one study to another. This may be referred to genetic \&racial causes or may be due to the different methodologies of sample collection and sample size.

The relation of anatomical variation to the incidence of paranasal sinusitis is debated.

In our study, $85.3 \%$ of patients have sinusitis, which is a high incidence supporting the possible correlation between anatomical variation and sinusitis. Nasal septum deviation is the most common of all (50\%). We find a correlation between nasal septum deviation and sporadic unclassified sinusitis $(\mathrm{P}=0.047)$. Our results are supported with those of Sarna A et al, 2002 who stated that "severe nasal septum deviation has been noted as a contributing factor for sinusitis" [10]. Significant bowing of the nasal septum results in narrowing of the middle meatus. Sometimes there may be attached nasal spurs which may further compromise the osteomeatal unit with increased incidence of developing sinusitis. However, the effect of nasal septum deviation or isolated nasal spur on the occurrence of sinusitis was not proved $[8,11]$. The reported prevalence of aeration of the middle turbinate varies widely from 14-80\% [12]. The concha bullosa in itself does not require surgery, but its presence may predispose a patient to occlusion of the ostiomeatal complex and subsequent sinus disease [13]. Concha bullosa is found in $23 \%$ of our participants with non-significant correlation between it and different types of sinusitis. The reported incidence of uncinate process variation ranges from 0.4 to $18 \%$ [9]. Uncinate process may show pneumatization, deviation or even absence. Pneumatization of the uncinate process is believed to be due to the extension of the agger nasi cell within the anterosuperior portion of the uncinate process. It has also been suggested as a predisposing factor for impaired ventilation of the anterior group of sinuses and frontal sinus. It may also change the normal anatomy of Osteomeatal complex thus proper drainage of sinuses causing sinusitis [13]. Only $6 \%$ in the current study have uncinate process variations, with significant correlation to unclassified sinusitis, including nonspecific mucosal thickening and mucocele (with the $p$ value $=0.024$ ).

In summary, anatomical variations of the nose and PNS are common and variable. They may be considered as contributing factors for the occurrence of inflammatory diseases of the PNS. However this correlation is still under investigation and needs more expanded researches with large sample size.

\section{Conclusion}

The commonest anatomical variation of the study population is nasal septal deviation $(50 \%)$, and the most common pattern of sinusitis is the infundibular pattern $(28 \%)$. There is significant correlation between nasal septal deviation, uncinate prosess anomalies to paranasal sinusitis ( $\mathrm{p}$ value $<0.05$ for each). CT scan can diagnose different types of anatomical variations of the nose and PNS owing to its high resolution, thin cuts, and reconstructed images. The importance of diagnosing such anomalies is not only in the correlation between their presence and patient's liability to sinus inflammatory diseases, but also in planning for surgical or endoscopic intervention of the PNS.

\section{References}

[1] Dwivedi A, Singha K. CT of the paranasal sinuses: normal anatomy, variants and pathology. Journal of Optoelectronics and Biomedical Materials 2010; 2: 289 - 281.

[2] Zinreich S, Albayram S, Benson M, Oliverio P. The ostiomeatal complex and functional endoscopic surgery. In: Peter MS, Hugh DC, eds. Head and Neck Imaging. St. Louis: Mosby; 2003: 149-74.

[3] Naimi M, Bakhshaei M. The major obstructive inflammatory patterns of the sinonasal diseases in 200 candidates of functional endoscopic sinus surgery. The Iranian Journal of Otorhinolaryngology 2006; 17, 42: 14-9.

[4] Azila A, Irfan M, Rohaizan Y, Shamim A K. The Prevalence of Anatomical Variations in Osteomeatal Unit in Patients with Chronic Rhinosinusitis. Med J Malaysia 2011; 66 (3):191-4.

[5] Fadda G, Rosso S, Aversa S, Petrelli A, Ondolo C, Succo G. Multiparametric statistical correlations between paranasal sinus anatomic variations and chronic rhinosinusitis. Acta Otorhinolaryngol Ital 2012; 32(4): 244-251.

[6] Kaygusuz A, Hakesever M, Akduman D, Aslan S, Sayar Z. Sinonasal anatomical variations: their relationship with chronic rhinosinusitis and effect on the severity of disease-a computerized tomography assisted anatomical and clinical study. Indian j otolaryngol head neck surg 2014;66(3):260-6. doi: 10.1007/s12070-013-0678-y. Epub 2013 Oct 15.

[7] Babbel WR, Harsnberger HR, Sonkens J. Recurring patterns of inflammatory sinonasal disease demonstrated on screening sinus CT. Seminar in U/S, CT and MRI 1991; 12 (6) : 511-519. 

Structures and Chronic Sinusitis by Computed Tomography

[8] Raut A, Jankharia B. Paranasal Sinuses in Health and Disease. Rhinology and Facial Plastic Surgery. Springer Berlin Heidelberg; 2009, P. 62-35.

[9] Adeel M, Rajput M, Akhter S, Ikram M, Arain A, Khattak Y. Anatomical variations of nose and para-nasal sinuses. CT scan review2013; 63(3):317-9.

[10] Sarna A, Hayman LA, Laine FJ, Taber KH. Coronal imaging of the osteomeatal unit: anatomy of 24 variants. J Comput Assist Tomogr 2002; 26(1):153-7.

[11] Stallman JS, Lobo JN, Som PM. The incidence of concha bullosa and its relationship to nasal septal deviations and paranasal sinus disease. Am J Neuroradiol 2004; 25:16131618.

[12] Miranda C, Maranho C, Arraes F, Padilha I, Farias L, Jatob M, et al. Anatomical variations of paranasal sinuses at multislice computed tomography: what to look for. Radiol Bras 2011; 44(4): 256-262.

[13] Al-Qudah M A. Anatomical Variations in Sino-Nasal Region: A Computer Tomography (CT) Study. J Med J 2010; Vol. 44 (3): 290-297 